\title{
Horizontal Factorizations of Certain Hasse-Weil Zeta Functions - a Remark on a Paper by Taniyama
}

\author{
Christopher Deninger (*) - Dimitri Wegner (*) \\ Dedicated to Francesco Baldassarri
}

\section{Introduction}

The paper [T] by Taniyama contains several results in the arithmetic theory of $C M$ abelian varieties which have become well known. Moreover the notion of a compatible system $\rho=\left(\rho_{l}\right)$ of $l$-adic representations is introduced there for the first time. Under suitable conditions on $\rho$, Taniyama proves an interesting formula for the alternating product of the $L$-functions of the exterior powers of $\rho$ : it is given by an infinite product of Artin $L$-functions each of which modified by a change of finitely many Euler factors. As an application, Taniyama obtains such a formula for the HasseWeil zeta function of an abelian scheme over a localized number ring.

These infinite product formulas of Taniyama seem to be little known but they motivated the works [JY] and [JR]. In [JY] Joshi and Yogananda show that the Hasse-Weil zeta function $\zeta_{\mathrm{G}_{m}}(s)=\zeta(s-1) / \zeta(s)$ of $\mathrm{G}_{m}=\operatorname{spec} Z\left[T, T^{-1}\right]$ is an infinite product of Dirichlet $L$-series. This is a special case of Taniyama's formulas but they give an elementary direct argument. In [JR] Joshi and Raghunathan express quotients of very general Dirichlet series with Euler products as infinite products of "twisted Dirichlet series". The method is purely local. It applies in particular to the Hasse-Weil zeta function of $\mathfrak{X} \times \mathrm{G}_{m}$ where $\mathfrak{X}$ is a scheme of finite type over Z. In this case the formula has a geometric proof which was suggested by Serre, c.f. [JR] § 3. Joshi and Raghunathan also obtain certain infinite product formulas for quotients of automorphic $L$-functions. Recently these have been related to canonical bases by Kim and Lee, [JY] Remark 2.21.

(*) Indirizzo degli A.: Mathematical Institute University of Münster Einsteinstr. 6248149 Münster, Germany.

E-mail: deninger@uni-muenster.deＥ-mail: dimwegner@uni-muenster.de 
In the present note we interpret Taniyama's identity for the HasseWeil zeta function of an abelian scheme $\mathcal{A}$ as a "horizontal factorization": We write $\mathcal{A}$ as an essentially disjoint union of horizontal prime divisors corresponding to certain closed points $P$ on the generic fibre $A=\mathcal{A} \otimes \mathrm{Q}$. The residue field of each point $P$ is a number field $K=K(P)$ and $\zeta_{\mathcal{A}}(s)$ is essentially the product of the corresponding Dedekind zeta-functions $\zeta_{K}(s)$. To get an exact formula one needs to change each $\zeta_{K}(s)$ at finitely many Euler factors. Our method is similar to the one suggested by Serre and used in [JR] $\S 3$ and surely our interpretation of Taniyama's formula is known to Serre. We found it before learning about [JR].

Section 2 contains a review of Taniyama's formula in the context of $l$ adic representations and a discussion of the examples coming from the $l$ adic cohomologies of $\mathrm{G}_{m}$ and abelian varieties.

In section 3 we establish the "horizontal factorization" of the HasseWeil zeta functions of a class of group schemes $\mathcal{G}$ containing all extensions of abelian schemes by Tori. The method is geometric and elementary. For $\mathrm{G}_{m}$ and abelian schemes the factorization formulas are the same as the ones in section 2 which follow from Taniyama's theorem. This clarifies the meaning of Taniyama's formulas in these cases.

In section 4 we give some generalizations. In particular there are "horizontal factorizations" for the zeta functions of arbitrary open subschemes in $\mathbb{P}^{N}$ over spec Z.

\section{Taniyama's product formula}

We begin by recalling a result of Taniyama using the terminology of [S] Ch. I which developed from [T]. Let $K / Q$ be an algebraic number field with ring of integers $\mathfrak{D}_{K}$ and absolute Galois group $G_{K}=\operatorname{Gal}(\bar{K} / K)$. We fix algebraic closures and embeddings $\overline{\mathrm{Q}} \subset \mathrm{C}$ and $\overline{\mathrm{Q}} \subset \overline{\mathrm{Q}}_{l}$ for each prime number $l$. Consider a strictly compatible system of integral $l$-adic representations $\rho=\left(\rho_{l}\right)$ of $G_{K}$ on free $Z_{l-}$ modules $E_{l}$ of rank $d \geq 1$. Let $S$ be the exceptional set of $\rho$. It is the smallest set of places of $K$ such that for $\mathfrak{p} \notin S$ the representation $\rho_{l}$ is unramified at $\mathfrak{p}$ for each $\mathfrak{p} \nmid l$. The set $S$ is finite. For a finite place $\mathfrak{p}$ of $K$ and a place $\mathfrak{B}$ of $\bar{K}$ extending $\mathfrak{p}$ let $I_{\mathfrak{B}} \subset G_{\mathfrak{\beta}} \subset G_{K}$ be the inertia and decomposition groups at $\mathfrak{P}$ and denote by $F_{\Re} \in G_{\Re} / I_{\mathfrak{s}}$ the (arithmetic) Frobenius element. If $\mathfrak{p} \notin S, \mathfrak{p} \nmid l$ the automorphism $F_{\mathfrak{*}, \rho_{l}}=$ $\rho_{l}\left(F_{\mathfrak{s}}\right)$ of $E_{l}$ is well defined. Let $F_{\mathfrak{p}, \rho_{l}}$ be its conjugacy class in Aut $E_{l}$. 
By assumption,

$$
P_{\mathfrak{p}, \rho}(T)=\operatorname{det}\left(1-F_{\mathfrak{p}, \rho_{l}} T \mid E_{l}\right) \quad \text { and } \quad Q_{\mathfrak{p}, \rho}(T)=\operatorname{det}\left(F_{\mathfrak{p}, \rho_{l}}-T \mid E_{l}\right)
$$

are polynomials of degree $d$ in Z $[T]$ which do not depend on $l$. It follows that $\operatorname{det} F_{\mathfrak{p}, \rho_{l}}$ is an integer independent of $l$. In this situation, Taniyama makes further assumptions:

(I) There is a non-negative integer $w$ independent of $\mathfrak{p} \notin S$ such that all zeroes of $Q_{\mathfrak{p}, \rho}(T)$ in C have absolute value $N \mathfrak{p}^{w / 2}$.

(II) $\operatorname{det} F_{\mathfrak{p}, \rho_{l}}$ is positive i.e. $P_{\mathfrak{p}, \rho}(T)=(-1)^{d} a_{d} T^{d}+\ldots+1$ with $a_{d}>0$ for any $\mathfrak{p} \notin S$.

(III) For $\mathfrak{p} \notin S$ with $\mathfrak{p} \mid l$ and any place $\mathfrak{B} \mid \mathfrak{p}$ view $E_{l}$ as a $G_{\mathfrak{x}}$-module. Then there are $l$-adic representations

$$
\rho_{l}^{0}: G_{\Re} \rightarrow \operatorname{Aut}\left(E_{l}^{0}\right) \quad \text { and } \quad \rho_{l}^{\text {ét }}: G_{\Re} \rightarrow \operatorname{Aut}\left(E_{l}^{\text {ét }}\right)
$$

on free $Z_{l}$-modules $E_{l}^{0}$ and $E_{l}^{\text {ét }}$ with the following properties:

(1) There is an exact sequence of $G_{\mathfrak{*}}$-modules

$$
0 \longrightarrow E_{l}^{0} \longrightarrow E_{l} \longrightarrow E_{l}^{\text {ét }} \longrightarrow 0 \text {. }
$$

(2) The representation $\rho_{l}^{\text {ét }}$ of $G_{\Re}$ is unramified.

(3) The eigenvalues of $\rho_{l}^{\text {et }}\left(F_{\Re \mathfrak{\beta}}\right)$ in $\bar{Q}_{l}$ are exactly those zeroes of $Q_{\mathfrak{p}, \rho}(T)$ under the fixed embedding $\bar{Q} \subset \bar{Q}_{l}$ which are $l$-adic units.

The representation spaces $E_{l}^{0}$ and $E_{l}^{\text {ét }}$ depend on $\mathfrak{p}$. In particular their ranks may vary with $\mathfrak{p}$. Condition (III) needs to be checked for one place $\mathfrak{P} \mid \mathfrak{p}$ only.

Consider the $L$-function of $\rho$

$$
L(\rho, s)=\prod_{\mathfrak{p} \notin S} P_{\mathfrak{p}, \rho}\left(N \mathfrak{p}^{-s}\right)^{-1} .
$$

By assumption it converges locally uniformly in $\operatorname{Re} s>1+\frac{w}{2}$. More generally, the $L$-function of $\Lambda^{i} \rho$ converges in $\operatorname{Re} s>1+\frac{i w}{2}$. In the theorem recalled below, Taniyama expresses the alternating product of the $L$-series $L\left(\Lambda^{i} \rho, s\right)$ as an infinite product of modified $\operatorname{Artin} L$-series. They are defined as follows: Consider the free $\hat{\mathrm{Z}}$-module $E=\prod E_{l}$ of rank $d$. It is a module under the Galois group $G_{K}$. For a place $\mathfrak{p} \notin S$ of $K$ and a place $\mathfrak{i} \mid \mathfrak{p}$ we also 
need the $\hat{Z}$-module

$$
E_{\mathfrak{p}}=\prod_{\mathfrak{p} \nmid l} E_{l} \times \prod_{\mathfrak{p} \mid l} E_{l}^{\text {ét }}
$$

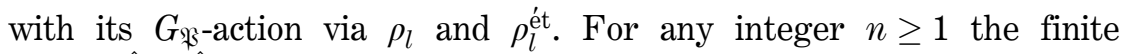
$\mathrm{Z} / n=\hat{\mathrm{Z}} / n \hat{\mathrm{Z}}$-modules $E(n)=E / n E$ and $E_{\mathfrak{p}}(n)=E_{\mathfrak{p}} / n E_{\mathfrak{p}}$ carry $G_{K^{-}}$resp. $G_{\mathfrak{p}}$-operations and for $\mathfrak{p} \nmid n$ we have $E_{\mathfrak{p}}(n)=E(n)$. By $E_{\mathfrak{p}}(n)^{*}$ and $E(n)^{*}$ we denote the subsets of elements of order $n$ in $E_{\mathfrak{p}}(n)$ resp. $E(n)$. They are $G_{\mathfrak{B}^{-}}$ resp. $G_{K}$-invariant. For $\mathfrak{p} \notin S$ and $v \geq 1$, Taniyama defines

$$
\psi_{n}\left(\mathfrak{p}^{v}\right):=\operatorname{Tr}\left(F_{\mathfrak{k}}^{v} \mid \mathrm{C}\left[E_{\mathfrak{p}}(n)^{*}\right]\right) .
$$

This is independent of the choice of $\mathfrak{P} \mid \mathfrak{p}$ and it follows from the Chinese remainder theorem that for coprime $n, m$ we have

$$
\psi_{n m}\left(\mathfrak{p}^{v}\right)=\psi_{n}\left(\mathfrak{p}^{v}\right) \psi_{m}\left(\mathfrak{p}^{v}\right) \quad \text { if } \mathfrak{p} \notin S .
$$

We may also consider the Artin representation of $G_{K}$ on $\mathrm{C}\left[E(n)^{*}\right]$. For its character we have in particular:

$$
\varphi_{n}\left(\mathfrak{p}^{v}\right)=\operatorname{Tr}\left(F_{\mathfrak{s}}^{v} \mid \mathrm{C}\left[E(n)^{*}\right]\right) .
$$

This shows:

$$
\psi_{n}\left(\mathfrak{p}^{v}\right)=\varphi_{n}\left(\mathfrak{p}^{v}\right) \quad \text { for all } \mathfrak{p} \notin S \text { with } \mathfrak{p} \nmid n .
$$

The $L$-function of $\psi_{n}$ is defined by the formula

$$
\begin{aligned}
L\left(\psi_{n}, s\right) & =\exp \sum_{\mathfrak{p} \notin S} \sum_{v=1}^{\infty} \frac{\psi_{n}\left(\mathfrak{p}^{v}\right)}{v} N \mathfrak{p}^{-v s} \\
& =\prod_{\mathfrak{p} \notin S} \operatorname{det}\left(1-F_{\mathfrak{s}} N \mathfrak{p}^{-s} \mid \mathrm{C}\left[E_{\mathfrak{p}}(n)^{*}\right]\right)^{-1} .
\end{aligned}
$$

The Artin $L$-function of $\varphi_{n}$ is given by the formula

$$
\begin{aligned}
L\left(\varphi_{n}, s\right) & =\exp \sum_{\mathfrak{p} \notin S} \sum_{v=1}^{\infty} \frac{\varphi_{n}\left(\mathfrak{p}^{v}\right)}{v} N \mathfrak{p}^{-v s} \\
& =\prod_{\mathfrak{p} \notin S} \operatorname{det}\left(1-F_{\mathfrak{s}} N \mathfrak{p}^{-s} \mid \mathrm{C}\left[E(n)^{*}\right]\right)^{-1} .
\end{aligned}
$$

The Euler-factors of $L\left(\psi_{n}, s\right)$ and $L\left(\varphi_{n}, s\right)$ are the same for all $\mathfrak{p} \notin S, \mathfrak{p} \nmid n$. Hence $L\left(\psi_{n}, s\right)$ is an Artin $L$-series up to finitely many Euler factors. Taniyama's result is the following, [T] Theorem 3. For the reader's convenience we sketch a proof. 
THEOREM (Taniyama). Under the previous assumptions the following equality holds in $\operatorname{Re} s>1+\frac{d w}{2}$

$$
\prod_{i=0}^{d} L\left(\Lambda^{i} \rho, s\right)^{(-1)^{d-i}}=\prod_{n=1}^{\infty} L\left(\psi_{n}, s\right) .
$$

Proof. Taking logarithmic derivatives on both sides we are reduced to showing that for any $\mathfrak{p} \notin S$ we have an equality where the sum on the right is finite

$$
\prod_{i=1}^{d}\left(\lambda_{i}^{v}-1\right)=\sum_{n=1}^{\infty} \psi_{n}\left(\mathfrak{p}^{v}\right) \quad \text { for } v \geq 1 .
$$

Here $\lambda_{1}, \ldots, \lambda_{d}$ are the zeroes of $Q_{\mathfrak{p}, \rho}(T)$. Since $\psi_{n}\left(\mathfrak{p}^{v}\right)$ is multiplicative in $n$ we need to see that

$$
\prod_{i=1}^{d}\left(\lambda_{i}^{v}-1\right)=\prod_{l} \sum_{k=0}^{\infty} \psi_{l^{k}}\left(\mathfrak{p}^{v}\right) .
$$

Here the sums on the right should be finite and equal to 1 for almost all $l$. The left hand side is in $Z$ and an elementary argument using assumptions (I) and (II) shows that it is positive. As for the right hand side, if $\mathfrak{p} \nmid l$ we have a commutative diagram

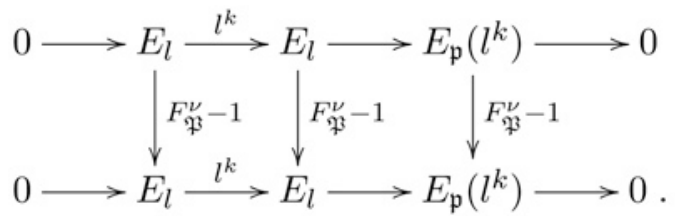

By assumption (I) the middle and left vertical maps are injective with finite cokernels of $l$-power order. The snake lemma gives an isomorphism

$$
\operatorname{Ker}\left(F_{\mathfrak{s}}^{v}-1 \mid E_{\mathfrak{p}}\left(l^{k}\right)\right) \stackrel{\sim}{\longrightarrow} \operatorname{Coker}\left(F_{\mathfrak{s}}^{v}-1 \mid E_{l}\right)_{l^{k}} .
$$

By definition, $\psi_{l^{k}}\left(\mathfrak{p}^{v}\right)$ is the number of elements of order $l^{k}$ in the group on the left. Hence $\psi_{l^{k}}\left(\mathfrak{p}^{v}\right)=0$ for large $k$ and we have:

$$
\begin{aligned}
\sum_{k=0}^{\infty} \psi_{l^{k}}\left(\mathfrak{p}^{v}\right) & =\left|\operatorname{Coker}\left(F_{\mathfrak{B}}^{v}-1 \mid E_{l}\right)\right| \\
& =\left|\operatorname{det}\left(F_{\mathfrak{B}}^{v}-1 \mid E_{l}\right)\right|_{l}^{-1} \\
& =\left|\prod_{i=1}^{d}\left(\lambda_{i}^{v}-1\right)\right|_{l}^{-1} .
\end{aligned}
$$


For prime numbers $l$ with $\mathfrak{p} \mid l$ we argue similarly replacing $E_{l}$ by $E_{l}^{\text {ét }}$. This gives

$$
\sum_{k=0}^{\infty} \psi_{l^{k}}\left(\mathfrak{p}^{v}\right)=\left|\operatorname{det}\left(F_{\mathfrak{s}}^{v}-1 \mid E_{l}^{\mathrm{e} t}\right)\right|_{l}^{-1}
$$

By assumption (III 3) the eigenvalues $\lambda_{i}$ which do not occur among the eigenvalues of $F_{\mathfrak{s}}$ on $E_{l}^{\text {ét }}$ are those with positive $l$-valuation. For them $\lambda_{i}^{v}-1$ is an $l$-adic unit and therefore we have

$$
\sum_{k=0}^{\infty} \psi_{l^{k}}\left(\mathfrak{p}^{v}\right)=\left|\prod_{i=1}^{d}\left(\lambda_{i}^{v}-1\right)\right|_{l}^{-1}
$$

in the case $\mathfrak{p} \mid l$ as well. Again the sum is finite. Equation (4) now follows from the product formula.

In order to understand Taniyama's formula better, note that the Artin $L$-series $L\left(\varphi_{n}, s\right)$ is a product of finitely many Dedekind zeta-functions: Decompose the $G_{K}$-set $E(n)^{*}$ into orbits

$$
E(n)^{*}=\coprod_{P} G_{K} P
$$

where $P$ runs over a set of representatives in $E(n)^{*}$ of the $G_{K}$-orbits. Let $K_{P}$ be the fixed field of the stabilizer of $P$ in $G_{K}$ i.e. $G_{K_{P}}=\left(G_{K}\right)_{P}$. Then we have

$$
\begin{aligned}
\mathbb{C}\left[E(n)^{*}\right] & =\bigoplus_{P} \mathbb{C}\left[G_{K} P\right]=\bigoplus_{P} \mathbb{C}\left[G_{K} / G_{K_{P}}\right] \\
& =\bigoplus_{P} \operatorname{Ind}_{G_{K_{P}}}^{G_{K}}(\mathbf{1}) .
\end{aligned}
$$

The formalism of Artin $L$-series [N] Ch. 7, $\S 10$ now implies the formula

$$
L\left(\varphi_{n}, s\right)=\prod_{P} \zeta_{K_{P}, S}(s)
$$

Here $\zeta_{K_{P}, S}(s)$ is the Dedekind zeta function of $K_{P}$ without the Euler factors for primes above $S$.

We now discuss the cyclotomic character and following Taniyama also the representation of $G_{K}$ on the Tate-module of an abelian variety.

The group $G_{K}$ acts on $T_{l} \mathbb{G}_{m}=\lim _{k} \mu_{l^{k}}(\bar{K})$. The corresponding representations $\rho_{l}: G_{K} \rightarrow \mathrm{GL}_{1}\left(T_{l} \mathrm{G}_{m}\right)=Z_{l}^{\times}$form a strictly compatible system $\rho=\left(\rho_{l}\right)$ with empty exceptional set $S$. We have $P_{\mathfrak{p}, \rho}(T)=1-N \mathfrak{p} T$. 
Hence conditions (I) and (II) are satisfied with $d=1, w=2$. Condition (III) holds with $E_{l}^{\text {et }}=0$ and $E_{l}^{0}=T_{l} \mathrm{G}_{m}$. The $L$-function of $\rho$ is $L(\rho, s)=\zeta_{K}(s-1)$. We have $E_{\mathfrak{p}}=\prod_{\mathfrak{p} \backslash l} T_{l} \mathrm{G}_{m}$ and hence $E_{\mathfrak{p}}(n)=\mu_{n_{\mathfrak{p}}}(\bar{K})$ where $n_{\mathfrak{p}}$ is the biggest prime to $\mathfrak{p}$ factor of $n$. It follows that $E_{\mathfrak{p}}(n)^{*}=\emptyset$ hence $\psi_{n}\left(\mathfrak{p}^{v}\right)=0$ if $\mathfrak{p} \mid n$ and that $E_{\mathfrak{p}}(n)^{*}$ is the set of primitive $n$-th roots of unity in $\bar{K}$ if $\mathfrak{p} \nmid n$.

For $\mathfrak{p} \nmid n$ choosing a primitive $n$-th root of unity $\zeta_{n}$, the map $v \mapsto \zeta_{n}^{v}$ gives a bijection $(Z / n)^{\times} \stackrel{\sim}{\longrightarrow} E_{\mathfrak{p}}(n)^{*}$. Multiplication by $N \mathfrak{p}$ on the left corresponds to the operation by $F_{\mathfrak{B}}$ on the right. It follows that

$$
\begin{aligned}
\psi_{n}\left(\mathfrak{p}^{v}\right) & =\operatorname{Tr}\left(F_{\mathfrak{p}}^{v} \mid \mathbb{C}\left[E_{\mathfrak{p}}(n)^{*}\right]\right) \\
& =\operatorname{Tr}\left(N \mathfrak{p}^{v} \mid \mathbb{C}\left[(Z / n)^{\times}\right]\right) \\
& =\sum_{\chi \in\left(\mathrm{Z}_{\mathrm{l} / n)^{\times}}\right.} \chi\left(N \mathfrak{p}^{v}\right) .
\end{aligned}
$$

Here we have used the decomposition of the regular representation of $(Z / n)^{\times}$into the direct sum of the characters $\chi$ of $(Z / n)^{\times}$. Setting

$$
L_{K}(\chi, s)=\prod_{\mathfrak{p} \nmid n}\left(1-\chi(N \mathfrak{p}) N \mathfrak{p}^{-s}\right)^{-1}
$$

we therefore find the formula

$$
L\left(\psi_{n}, s\right)=\prod_{\chi \in(\bar{Z} / n)^{\times}} L_{K}(\chi, s) .
$$

Taniyama's theorem therefore implies the following relation:

$$
\zeta_{K}(s-1) / \zeta_{K}(s)=\prod_{n=1}^{\infty} \prod_{\chi \in(\bar{Z} / n)^{x}} L_{K}(\chi, s) \quad \text { in } \operatorname{Re} s>2 .
$$

For $K=$ Q, a direct proof of this formula is given in [JY]. We can make formula (5) more explicit in the example. The set $E(n)$ is the set of $n$-th roots of unity. The $G_{K}$-orbits on $E(n)$ are in bijection with the set $\left|\mu_{n, K}\right|$ of closed points $P$ of the finite group scheme $\mu_{n, K}=\operatorname{spec} K[T] /\left(T^{n}-1\right)$ and $K_{P}$ is the residue field of $P$, a finite extension of $K$. Thus the $G_{K}$-orbits on $E(n)^{*}$ are in bijection with

$$
\left|\mu_{n, K}^{*}\right|:=\left|\mu_{n, K}\right| \backslash \bigcup_{\substack{d \mid n \\ d \neq n}}\left|\mu_{d, K}\right| .
$$

According to (5), we have

$$
L\left(\varphi_{n}, s\right)=\prod_{P \in\left|\mu_{n, K}^{*}\right|} \zeta_{K_{P}}(s) .
$$


The $L$-functions $L\left(\varphi_{n}, s\right)$ and $L\left(\psi_{n}, s\right)$ have the same Euler factors for $\mathfrak{p} \nmid n$. For $\mathfrak{p} \mid n$ the Euler factor of $L\left(\psi_{n}, s\right)$ equals 1 . Accordingly we modify $\zeta_{K_{P}}(s)$ for $P \in\left|\mu_{n, K}^{*}\right|$ by removing the Euler factors for primes dividing $n$. Let $\tilde{\zeta}_{K_{P}}(s)$ be the resulting zeta function

$$
\tilde{\zeta}_{K_{P}}(s)=\prod_{\mathfrak{q} \backslash n}\left(1-N \mathfrak{q}^{-s}\right)^{-1}
$$

where $q$ runs over the prime ideals of the ring of integers in $K_{P}$. Then (9) implies the formula

$$
L\left(\psi_{n}, s\right)=\prod_{P \in\left|\mu_{n, K}^{*}\right|} \tilde{\zeta}_{K_{P}}(s)
$$

Using (8) and the relation $\zeta_{\mathrm{G}_{m, \mathcal{O}_{K}}}(s)=\zeta_{K}(s-1) / \zeta_{K}(s)$ we get

$$
\zeta_{\mathrm{G}_{m, 0_{K}}}(s)=\prod_{P} \tilde{\zeta}_{K_{P}}(s) .
$$

Here $P$ runs over the closed points of $\mathrm{G}_{m, K}$ which are torsion i.e. correspond to Galois orbits of torsion points in $\mathrm{G}_{m, K}(\bar{K})$.

The second example, due to Taniyama in [T] section 18 will be discussed more briefly. Let $A / K$ be an abelian variety of dimension $g \geq 1$ and let $S$ be the set of places where $A$ has bad reduction. Let $\mathcal{A}$ be the extension of $A$ to an abelian scheme over spec $\mathfrak{D}_{K} \backslash S$. Then $S$ is the exceptional set of the strictly compatible system $\rho=\left(\rho_{l}\right)$ where $\rho_{l}$ is the representation of $G_{K}$ on the Tate module $T_{l} A=\lim _{k} A_{l^{k}}(\bar{K})$. Conditions (I) and (II) are known to be true with $d=2 g$ and $w=1$ by the work of Weil [We].

As for condition (III) let $K_{\mathfrak{p}}$ be the completion of $K$ at the place $\mathfrak{p}$. Let $\bar{K}_{\mathfrak{p}}$ be the algebraic closure of $K_{\mathfrak{p}}$ in the completion of $\bar{K}$ with respect to a given place $\mathfrak{P}$ of $\bar{K}$ above $\mathfrak{p}$. We write $\mathfrak{D}_{\mathfrak{p}}$ and $\overline{\mathfrak{D}}_{\mathfrak{p}}$ for the respective rings of integers. The residue field $k_{\mathfrak{p}}=\mathfrak{D}_{K} / \mathfrak{p}=\mathfrak{D}_{\mathfrak{p}} / \mathfrak{p D}_{\mathfrak{p}}$ has $N \mathfrak{p}$ elements and $\bar{k}_{\mathfrak{p}}=\overline{\mathfrak{D}}_{\mathfrak{p}} / \mathfrak{P} \overline{\mathfrak{D}}_{\mathfrak{p}}$ is an algebraic closure of $k_{\mathfrak{p}}$. Now assume that $\mathfrak{p} \notin S$ and $\mathfrak{p} \mid l$. The connected-étale exact sequence of the $l$-divisible group $\mathcal{A}(l)$ of $\mathcal{A}$ over $\mathfrak{b}_{\mathfrak{p}}$

$$
0 \longrightarrow \mathcal{A}(l)^{0} \longrightarrow \mathcal{A}(l) \longrightarrow \mathcal{A}(l)^{\mathrm{e} t} \longrightarrow 0
$$

gives an exact sequence of Tate-modules with $G_{\mathfrak{p}}=\operatorname{Gal}\left(\bar{K}_{\mathfrak{p}} / K_{\mathfrak{p}}\right)$-action

$$
0 \longrightarrow T\left(\mathcal{A}(l)^{0}\right) \longrightarrow T_{l} A \longrightarrow T_{l}\left(\mathcal{A} \otimes k_{\mathfrak{p}}\right) \longrightarrow 0 .
$$


Setting $E_{l}^{0}=T\left(\mathcal{A}(l)^{0}\right)$ and

$$
E_{l}^{\text {ét }}=T_{l}\left(\mathcal{A} \otimes k_{\mathfrak{p}}\right)=\lim _{\leftarrow} \mathcal{A}\left(\bar{k}_{\mathfrak{p}}\right)_{l^{v}}
$$

the conditions in (III) are verified.

For a scheme $\mathfrak{X}$ of finite type over spec $Z$ there is the Hasse-Weil zeta function of $\mathfrak{X}$

$$
\zeta_{\mathfrak{X}}(s)=\prod_{x \in|\mathfrak{X}|}\left(1-N x^{-s}\right)^{-1} \quad \text { for } \operatorname{Re} s>\operatorname{dim} \mathfrak{X} .
$$

Here $|\mathfrak{X}|$ is the set of closed points of $\mathfrak{X}$ and for $x \in \mathfrak{X}$ the number of elements in the finite residue field $\kappa(x)$ is denoted by $N x$. Using the basic relation

$$
\zeta_{\mathcal{A}}(s)=\prod_{i=0}^{2 g} L\left(\Lambda^{i} \rho, s\right)^{(-1)^{i}}
$$

Taniyama's theorem gives the formula

$$
\zeta_{\mathcal{A}}(s)=\prod_{n=1}^{\infty} L\left(\psi_{n}, s\right)
$$

We do not work out $L\left(\psi_{n}, s\right)$ completely but only $L\left(\varphi_{n}, s\right)$ which has the same Euler factors for $\mathfrak{p} \nmid n$. We have $E(n)=A_{n}(\bar{K})$ and $E(n)^{*}$ is the subset of $A_{n}(\bar{K})$ of elements of order $n$. The $G_{K}$-orbits on $A_{n}(\bar{K})$ are in bijection with the closed points $P$ of $A_{n}$ and $K_{P}$ is the residue field of $P$. Set

$$
\left|A_{n}^{*}\right|=\left|A_{n}\right| \backslash \bigcup_{\substack{d \mid n \\ d \neq n}}\left|A_{d}\right|
$$

Then according to (5) we have the formula

$$
L\left(\varphi_{n}, s\right)=\prod_{P \in\left|A_{n}^{*}\right|} \zeta_{K_{P}, S}(s)
$$

For $P \in\left|A_{n}^{*}\right|$ let $\tilde{\zeta}_{K_{P}, S}(s)$ be modifications of $\zeta_{K_{P}, S}(s)$ at the Euler factors of primes $\mathfrak{p} \mid n$ such that we have

$$
L\left(\psi_{n}, s\right)=\prod_{P \in\left|A_{n}^{*}\right|} \tilde{\zeta}_{K_{P}, S}(s)
$$


Then Taniyama's formula becomes

$$
\zeta_{\mathcal{A}}(s)=\prod_{P} \tilde{\zeta}_{K_{P}, S}(s)
$$

Here $P$ runs over the closed torsion points of $A$.

\section{Horizontal factorizations for group schemes}

In this section we give a simple geometric proof of a generalization to certain commutative group schemes $\mathcal{G}$ of equations (11) and (14) which concerned $\mathbb{G}_{m}$ resp. $\mathcal{A}$. The set of closed points of $\mathcal{G}$ will be essentially partitioned into the set of closed points on the horizontal prime divisors in $\mathcal{G}$ obtained by taking the closures in $\mathcal{G}$ of torsion points $P$ on the generic fibre of $\mathcal{G}$.

For a finite set $S$ of maximal ideals in $\mathfrak{D}_{K}$ set $U=\operatorname{spec} \mathfrak{D}_{K} \backslash S$. Thus $U=\operatorname{spec} \mathfrak{D}_{K, S}$ where

$$
\mathfrak{D}_{K, S}=\left\{\frac{f}{g} \mid f, g \in \mathfrak{D}_{K}, \mathfrak{p} \backslash g \text { for } \mathfrak{p} \notin S\right\} .
$$

We consider commutative smooth separated group schemes $\mathcal{G}$ of finite type over $U$ for which the $n$-multiplication is finite and flat for all $n \geq 1$. For simplicity we call such group schemes "good". Thus $\mathbb{G}_{m}$ and $\mathcal{A}$ above are good but $\mathrm{G}_{a}$ is not. Every extension of an abelian scheme over $U$ by a torus is good. In fact there is the following statement which was suggested by the referee together with an outline of the proof:

Proposition 1. For a "good" group scheme $\mathcal{G}$ over $U$ with connected generic fibre $G=\mathcal{G} \otimes K$ there is an open subscheme $\emptyset \neq V \subset U$ such that $\mathcal{G}_{V}=\mathcal{G} \times_{U} V$ is an extension of an abelian scheme by a torus.

Proof. By assumption $G$ is a commutative, connected smooth algebraic group over the perfect field $K$. According to a theorem of Chevalley there is a canonical exact sequence of algebraic groups over $K$

$$
0 \longrightarrow H \longrightarrow G \stackrel{\pi_{K}}{\longrightarrow} A \longrightarrow 0 \text {. }
$$

Here $H$ is affine, connected and smooth and $A$ is an abelian variety. See [C], Theorem 1.1 for a modern proof of Chevalley's theorem. Now we apply the theory of [EGAIV3] $\S 8,9$ and in particular the remark at the end of 8.8. It 
follows that the sequence (15) extends to an exact sequence of commutative group schemes over an open subscheme $\emptyset \neq V \subset U$

$$
0 \longrightarrow \mathcal{H} \longrightarrow \mathcal{G}_{V} \stackrel{\pi}{\longrightarrow} \mathcal{A}
$$

with the following properties: The commutative group scheme $\mathcal{H}$ is smooth affine and of finite type over $V$ with connected fibres and $\mathcal{A}$ is an abelian scheme over $V$. Reasons: The extensions $\mathcal{H}$ and $\mathcal{A}$ of $H$ and $A$ to group schemes over a suitable $V$ may be assumed to have connected fibres because of [EGAIV3] Lemma (9.7.1). The properties "affine" of $H / K$ and "proper" of $A / K$ extend to $\mathcal{H} / V$ resp. $\mathcal{A} / V$ by [EGAIV3] Théorème (8.10.5). In order to see that $G \stackrel{\pi_{K}}{\longrightarrow} A$ extends to a faithfully flat morphism $\mathcal{G}_{V} \stackrel{\pi}{\longrightarrow} \mathcal{A}$ after shrinking $V$ we use [EGAIV3] Proposition (9.6.1). It implies that the set of points $\mathfrak{p} \in V$ where $\pi_{\mathfrak{p}}$ is surjective is constructible in $V$. Since it contains the generic point spec $K$ of $V$ it contains a non-empty open subset of $V$. We may therefore assume that $\pi: \mathcal{G}_{V} \rightarrow \mathcal{A}$ is surjective, and also flat by [EGAIV3] Théorème (11.2.6) since $\pi_{K}$ is flat. Thus we have an exact sequence of group schemes

$$
0 \longrightarrow \mathcal{H} \longrightarrow \mathcal{G}_{V} \longrightarrow \mathcal{A} \longrightarrow 0
$$

The structure theory of commutative affine connected algebraic groups implies that there is a $K$-isomorphism $H \cong T \times{ }_{K} H_{u}$ where $T$ is a torus over $K$ and $H_{u}$ a commutative unipotent algebraic group, c.f. [DG] IV $\S 3$ Théorème 1.1. If $H_{u}$ is non-zero $G$ contains $\mathbb{G}_{a, K}$ as a closed subgroup. As before this inclusion may be extended over an open subset of $V$. Hence there is a point $\mathfrak{p} \in V \subset U$ with $G_{a, k_{\mathfrak{p}}}$ a closed algebraic subgroup of $\mathcal{G}_{k_{\mathfrak{p}}}$. Let $n=$ char $k_{\mathfrak{p}}$. Then we have

$$
\mathrm{G}_{a, k_{\mathfrak{p}}}=\left(\mathbb{G}_{a, k_{\mathfrak{p}}}\right)_{n} \subset\left(\mathcal{G}_{k_{\mathfrak{p}}}\right)_{n} .
$$

This is a contradiction because for good $\mathcal{G}$ the group scheme $\mathcal{G}_{n}$ is finite for any $n \geq 1$. Hence $H_{u}=0$ and $H \cong T$ is a torus. Over a finite extension $L / K$ the torus $H \otimes_{K} L$ is isomorphic to $\mathbb{G}_{m, L}^{r}$. Arguing as before we see that possibly after shrinking $V$ there is a finite étale covering of $V$ over which $\mathcal{H}$ becomes isomorphic to a product of $\mathbb{G}_{m}$ 's. Hence $\mathcal{H}$ restricted to $V$ is a torus.

We now fix a "good" group scheme $\mathcal{G}$ over an open subscheme $U=\operatorname{spec} \mathfrak{D}_{K} \backslash S$ as above. Then $\mathcal{G}_{n}$ is a finite flat group scheme over $U$. For $\mathfrak{p} \in U$ the reduction $\mathcal{G}_{n, k_{\mathfrak{p}}}=\mathcal{G}_{n} \otimes k_{\mathfrak{p}}$ is an étale group scheme over $k_{\mathfrak{p}}$ if 
$\mathfrak{p} \nmid n$. For $\mathfrak{p} \mid n$ we have the connected-étale sequence

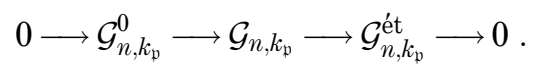

It splits since $\mathcal{G}_{n, k_{\mathrm{p}}}^{\text {red }}$ is a closed subgroup scheme of $\mathcal{G}_{n, k_{\mathfrak{p}}}$ because $k_{\mathfrak{p}}$ is perfect and the composition

$$
\mathcal{G}_{n, k_{\mathrm{p}}}^{\mathrm{red}} \hookrightarrow \mathcal{G}_{n, k_{\mathrm{p}}} \longrightarrow \mathcal{G}_{n, k_{\mathrm{p}}}^{\text {ét }}
$$

is an isomorphism, c.f. [Wa] 6.8. The unit section of $\mathcal{G}$ is a regular immersion by [L] 6 Proposition 3.13. Hence $\mathcal{G}_{n} \hookrightarrow \mathcal{G}$ is a regular immersion as well, the $n$-multiplication being flat. It follows that $\mathcal{G}_{n}$ is purely one-dimensional. See [L] 6 Proposition 3.11 for both assertions. The irreducible components of $\mathcal{G}_{n}$ are therefore the closures in $\mathcal{G}$ of the closed points $P$ of $\mathcal{G}_{n, K}=\mathcal{G}_{n} \otimes K$. Let $C_{P}=\{\bar{P}\}$ be the irreducible component of $\mathcal{G}_{n}$ corresponding to $P$ with its induced reduced structure. Over $U_{n}=U \backslash S_{n}$ with $S_{n}=\{\mathfrak{p} \mid n\}$ the scheme $\mathcal{G}_{n}$ is finite and étale. Hence the regular purely one-dimensional scheme $\mathcal{G}_{n} \times{ }_{U} U_{n}$ is the disjoint union of the restrictions of the $C_{P}$ to $U_{n}$

$$
\mathcal{G}_{n} \times_{U} U_{n}=\coprod_{P} C_{P} \times_{U} U_{n} .
$$

Let $K_{P}$ be the residue field of $P$, an algebraic number field. Since $C_{P} \rightarrow U$ is finite and $U=\operatorname{spec} \mathrm{D}_{K, S}$ is affine, $C_{P}=\operatorname{spec} A_{P}$ is affine as well. The ring $A_{P}$ is an order in $\mathfrak{D}_{K_{P}, S}$ and since $C_{P} \times_{U} U_{n}$ and hence $A_{P, S_{n}}$ is regular we have $\mathfrak{D}_{K_{P}, S \cup S_{n}}=A_{P, S_{n}}$. We therefore find

$$
C_{P} \times_{U} U_{n}=\operatorname{spec} \mathfrak{D}_{K_{P}, S \cup S_{n}} .
$$

Noting that $C_{P} \times_{U} U_{n}$ is even étale over $U_{n}$ it follows that the extension of number fields $K_{P} / K$ is unramified at all maximal ideals $\mathfrak{p} \notin S \cup S_{n}$.

The closed points of $\mathcal{G}$ are contained in the closed fibres $\mathcal{G}_{k_{\mathfrak{p}}}=\mathcal{G} \otimes k_{\mathfrak{p}}$ for maximal ideals $\mathfrak{p} \notin S$. A closed point $x$ of $\mathcal{G}_{k_{\mathfrak{p}}}$ corresponds to a finite $\operatorname{Gal}\left(\bar{k}_{\mathfrak{p}} / k_{\mathfrak{p}}\right)$-orbit in $\mathcal{G}_{k_{\mathfrak{p}}}\left(\bar{k}_{\mathfrak{p}}\right)=\mathcal{G}\left(\bar{k}_{\mathfrak{p}}\right)$. Since $k_{\mathfrak{p}}$ is finite, the group $\mathcal{G}\left(\bar{k}_{\mathfrak{p}}\right)$ is a union of finite abelian groups and hence every element has finite order. It follows that we have an equality

$$
|\mathcal{G}|=\bigcup_{n=1}^{\infty}\left|\mathcal{G}_{n}\right| .
$$

For any group scheme $\mathcal{H}$ over a scheme we denote by $\mathcal{H}_{n}^{*}$ the open subscheme of $\mathcal{H}_{n}$ of points of order $n$ :

$$
\mathcal{H}_{n}^{*}=\mathcal{H}_{n} \backslash \bigcup_{\substack{d \mid n \\ d \neq n}} \mathcal{H}_{d}
$$


With this notation we get the disjoint decomposition:

$$
|\mathcal{G}|=\coprod_{n=1}^{\infty}\left|\mathcal{G}_{n}^{*}\right|
$$

This implies the formula

$$
\zeta_{\mathcal{G}}(s)=\prod_{n=1}^{\infty} \zeta_{\mathcal{G}_{n}^{*}}(s)
$$

By equations (18) and (19) we have

$$
\mathcal{G}_{n}^{*} \times_{U} U_{n}=\coprod_{Q} \operatorname{spec} \mathfrak{D}_{K_{Q}, S \cup S_{n}}
$$

where $Q$ runs over the closed points of order $n$ in $\mathcal{G}_{K}$.

Hence

$$
\zeta_{\mathcal{G}_{n}^{*} \times U} U_{n}(s)=\prod_{Q} \zeta_{K_{Q}, S \cup S_{n}}(s)
$$

Changing the finitely many Euler factors at primes dividing $n$ suitably one gets modifications $\tilde{\zeta}_{K_{Q}, S}$ of $\zeta_{K_{Q}, S}$ for which the formula

$$
\zeta_{\mathcal{G}_{n}^{*}}(s)=\prod_{Q} \tilde{\zeta}_{K_{Q}, S}(s)
$$

holds. Then (20) becomes an equation

$$
\zeta_{\mathcal{G}}(s)=\prod_{P} \tilde{\zeta}_{K_{P}, S}(s)
$$

where $P$ runs over the closed torsion points of $\mathcal{G}_{K}$. This gives a simple geometric explanation for equation (14). To make these modifications more explicit we now discuss the zeta-function of $\mathcal{G}_{n}^{*}$ in more detail. In the decomposition

$$
\zeta_{\mathcal{G}_{n}^{*}}(s)=\prod_{\mathfrak{p} \notin S} \zeta_{\mathcal{G}_{n}^{*} \otimes k_{\mathfrak{p}}}(s)
$$

the factors at primes not dividing $n$ are understood by formula (22). Let now $\mathfrak{p} \notin S$ be a prime with $\mathfrak{p} \mid n$. We have $\mathcal{G}_{n}^{*} \otimes k_{\mathfrak{p}}=\left(\mathcal{G}_{n, k_{\mathfrak{p}}}\right)^{*}$. Since $k_{\mathfrak{p}}$ is perfect, $\mathcal{G}_{n, k_{p}}^{\text {red }}$ is a closed subgroup scheme of $\mathcal{G}_{n, k_{p}}$ and we have

$$
\left(\mathcal{G}_{n}^{*} \otimes k_{\mathfrak{p}}\right)^{\mathrm{red}}=\mathcal{G}_{n, k_{\mathfrak{p}}}^{\mathrm{red} *} \quad \text { and } \quad \mathcal{G}_{n, k_{\mathfrak{p}}}^{\text {red }} \cong \mathcal{G}_{n, k_{\mathfrak{p}}}^{\text {ét }}
$$


For the $\mathfrak{p}$-factor in equation (24) we therefore find

$$
\zeta_{\mathcal{G}_{n}^{*} \otimes k_{\mathrm{p}}}(s)=\zeta_{\mathcal{G}_{n, k_{\mathrm{p}}}^{\text {red }}}(s)=\zeta_{\mathcal{G}_{n, k_{\mathrm{p}}}^{\text {etex }}}(s) .
$$

Consider the connected-étale sequence for the group scheme $\mathcal{G}_{n, \mathfrak{v}_{\mathfrak{p}}}=$ $\mathcal{G}_{n} \otimes_{U} \mathfrak{D}_{\mathfrak{p}}$

$$
0 \longrightarrow \mathcal{G}_{n, \mathfrak{o}_{\mathfrak{p}}}^{0} \longrightarrow \mathcal{G}_{n, \mathfrak{o}_{\mathfrak{p}}} \longrightarrow \mathcal{G}_{n, \mathfrak{o}_{\mathfrak{p}}}^{\text {ét }} \longrightarrow 0 \text {. }
$$

The closed points of $\mathcal{G}_{n, k_{\mathrm{p}}}^{\text {et }}$ are in bijection with the closed points of $\mathcal{G}_{n, K_{\mathrm{p}}}^{\text {et }}=$ $\mathcal{G}_{n, \mathfrak{o}_{\mathfrak{p}}}^{\text {ét }} \otimes_{\mathfrak{p}_{\mathfrak{p}}} K_{\mathfrak{p}}$ because the Galois orbits on $\mathcal{G}_{n, \mathfrak{p}_{\mathfrak{p}}}^{\text {ét }}\left(\bar{K}_{\mathfrak{p}}\right)=\mathcal{G}_{n, \mathfrak{o}_{\mathfrak{p}}}^{\text {ét }}\left(\bar{k}_{\mathfrak{p}}\right)=\mathcal{G}_{n, k_{\mathfrak{p}}}^{\text {ét }}\left(\bar{k}_{\mathfrak{p}}\right)$ are the same. Hence the closed points of $\mathcal{G}_{n, k_{p}}^{\text {etw }}$ are in bijection with the closed points of $\mathcal{G}_{n, K_{\mathrm{p}}}^{\text {ét }}$ of order $n$. Therefore the finite product of Euler factors defining $\zeta_{\mathcal{G}_{n}^{*} \otimes k_{p}}(s)$ can also be described in terms of the generic fibre $\mathcal{G}_{n, K_{\mathrm{p}}}^{\text {ét }}$. For $\mathcal{G}=\mathcal{A}$ an abelian scheme over $U$ this is the geometry behind Taniyama's definition of the Euler factors of $L\left(\psi_{n}, s\right)$ over primes $\mathfrak{p}$ dividing $n$.

Example. Consider $\mathcal{G}=\mathrm{G}_{m, \mathfrak{o}_{K}}$ over $U=\operatorname{spec} \mathfrak{D}_{K}$ and thus $\mathcal{G}_{n}=\mu_{n, \mathfrak{o}_{K}}$. The fibres of $\mathcal{G}_{n}^{*}$ over primes $\mathfrak{p} \mid n$ are empty because in this case the order of $\mu_{n}\left(\bar{k}_{\mathfrak{p}}\right)$ is less than $n$ and so there are no points of order $n$. It follows that $\mathcal{G}_{n}^{*}=\mathcal{G}_{n}^{*} \times U_{n}$. By formula (22) we have

$$
\zeta_{\mathcal{G}_{n}^{*} \times U_{n}}(s)=\prod_{Q} \zeta_{K_{Q}, S_{n}}(s)
$$

where $Q$ runs over the closed points of order $n$ in $\mathrm{G}_{m, K}$. The zeta function $\zeta_{K_{Q}, S_{n}}(s)$ is the same as the modified Dedekind zeta function $\tilde{\zeta}_{K_{Q}}(s)$ obtained by removing all Euler factors in $\zeta_{K_{Q}}(s)$ over primes dividing $n$. It follows that we have

$$
\zeta_{\mathcal{G}_{n}^{*}}(s)=\prod_{Q} \tilde{\zeta}_{K_{Q}}(s)
$$

and therefore by (20) with $P$ running over the closed torsion points of $\mathrm{G}_{m, K}$

$$
\zeta_{\mathrm{G}_{m, 0_{K}}}(s)=\prod_{P} \tilde{\zeta}_{K_{P}}(s) .
$$

Thus we obtain equation (11) again. One can make $\mathcal{G}_{n}^{*}$ explicit as follows. Consider first the case $K=\mathrm{Q}$. The primitive roots of unity of order $n$ in $\overline{\mathrm{Q}}^{\times}$ are all conjugated by $G_{\mathrm{Q}}$. Hence $\mathcal{G}_{\mathrm{Q}}=\mathrm{G}_{m, \mathrm{Q}}$ has exactly one closed point $Q$ of order $n$. It corresponds to the maximal ideal generated by the cyclotomic 
polynomial $\Phi_{n}(T)$ in $\mathrm{Q}\left[T, T^{-1}\right]$. Its residue field is the cyclotomic field $Q\left(\zeta_{n}\right)$. Formula (21) therefore gives:

$$
\mu_{n, Z}^{*}=\mu_{n, Z}^{*} \times U_{n}=\operatorname{spec} Z\left[1 / n, \zeta_{n}\right]=\operatorname{spec} Z[1 / n][T] /\left(\Phi_{n}(T)\right) .
$$

The latter description for $\mu_{n, Z}^{*}$ is the one used in [JR] Example 3.1. Equation (29) also follows from the fact that $\Phi_{d}$ and $\Phi_{e}$ are coprime in $Z[1 / n][T]$ if $d \neq e$ and $d \mid n$ and $e \mid n$, use e.g. [A]. In any case, one has

$$
\zeta_{\mu_{n, Z}^{*}}(s)=\tilde{\zeta}_{\mathrm{Q}\left(\zeta_{n}\right)}(s)
$$

and therefore

$$
\zeta_{\mathrm{G}_{m, Z}}(s)=\prod_{n=1}^{\infty} \tilde{\zeta}_{\mathrm{Q}\left(\zeta_{n}\right)}(s) .
$$

In the general case base change gives

$$
\mu_{n, \mathfrak{o}_{K}}^{*}=\operatorname{spec} Z_{[}\left[1 / n, \zeta_{n}\right] \otimes_{\mathrm{Z}} \mathfrak{D}_{K}=\operatorname{spec} \mathfrak{D}_{K}[1 / n][T] /\left(\Phi_{n}(T)\right) .
$$

Thus the fibre over $K$ decomposes into finitely many points $Q$ corresponding to the irreducible divisors of $\Phi_{n}$ over $K$. We can form their modified Dedekind zeta functions $\tilde{\zeta}_{K_{Q}}(s)$ and from them obtain $\zeta_{\mathrm{G}_{m, 0_{K}}}(s)$ by formula (28).

\section{Generalizations}

For a scheme $\mathfrak{X}$ which is separated of finite type over $U=\operatorname{spec} \mathfrak{D}_{K} \backslash S$ and a group scheme $\mathcal{G} / U$ as before we have

$$
\left|\mathfrak{X} \times_{U} \mathcal{G}\right|=\coprod_{n=1}^{\infty}\left|\mathfrak{X} \times_{U} \mathcal{G}_{n}^{*}\right| .
$$

Hence

$$
\zeta_{\mathfrak{X} \times{ }_{U} \mathcal{G}}(s)=\prod_{n=1}^{\infty} \zeta_{\mathfrak{X} \times_{U} \mathcal{G}_{n}^{*}}(s) .
$$

Using the decomposition (21) we find

$$
\mathfrak{X} \times_{U} \mathcal{G}_{n}^{*} \times_{U} U_{n}=\coprod_{Q} \mathfrak{X} \otimes_{\mathfrak{o}_{K, S}} \mathfrak{D}_{K_{Q}, S \cup S_{n}} .
$$

Hence the zeta-function of $\mathfrak{X} \times_{U} \mathcal{G}_{n}^{*}$ is up to the Euler factors for $\mathfrak{p} \mid n$ equal to the product of the zeta functions of $\mathfrak{X} \otimes_{\mathfrak{D}_{K, S}} \mathfrak{D}_{K_{Q}, S \cup S_{n}}$. 
For $\mathcal{G}=\mathbb{G}_{m}$ over $U=\operatorname{spec} Z$ we have

$$
\mathfrak{X} \times \mu_{n, Z}^{*}=\mathfrak{X} \otimes \mathbb{Z}\left[1 / n, \zeta_{n}\right]
$$

and hence

$$
\zeta_{\mathfrak{X} \times \mathrm{G}_{m}}(s)=\prod_{n=1}^{\infty} \zeta_{\mathfrak{X} \otimes Z\left[1 / n, \zeta_{n}\right]}(s) .
$$

In [JR] section 3 the zeta function of $\mathfrak{X} \otimes \mathbb{Z}\left[1 / n, \zeta_{n}\right]$ is further decomposed into a product of zeta-functions of $\mathfrak{X}$ twisted by Dirichlet characters.

\section{More general schemes}

Consider an open subscheme $\mathcal{U} \subset \mathbb{P}^{N}=\mathbb{P}_{Z}^{N}$. We will describe a way to get a horizontal factorization of the zeta function of $\mathcal{U}$. Setting $\mathfrak{X}=\mathbb{P}^{N} \backslash \mathcal{U}$, the formula

$$
\zeta_{\mathfrak{X}}(s) \zeta_{\mathcal{U}}(s)=\zeta_{\mathbb{P}^{N}}(s)=\zeta(s) \zeta(s-1) \cdots \zeta(s-N)
$$

then gives information on the zeta function of the projective scheme $\mathfrak{X}$. Using the standard decomposition

$$
\mathbb{P}^{N}=\mathbb{A}^{N} \amalg \ldots \amalg \mathbb{A}^{0}
$$

we have

$$
\zeta_{\mathcal{U}}(s)=\prod_{M=0}^{N} \zeta_{\mathcal{U} \cap A^{M}}(s)
$$

For $I=\{0,1\}^{M}$ and $i \in I$ set $A_{i}=A_{i_{1}} \times \ldots \times A_{i_{M}}$ where $A_{0}=0_{\mathrm{A}^{M}}=$ spec $Z_{s}$ and $A_{1}=\mathbb{G}_{m}$. Then we have

$$
\mathrm{A}^{M}=\coprod_{i \in I} A_{i}
$$

and hence

$$
\zeta_{\mathcal{U} \cap A^{M}}(s)=\prod_{i \in I} \zeta_{\mathcal{U} \cap A_{i}}(s)
$$

Using the canonical isomorphism $A_{i}=\mathrm{G}_{m}^{|i|}$ where $|i|=i_{1}+\ldots+i_{M}$ we will identify $\mathcal{U} \cap A_{i}$ with an open subscheme of $\mathrm{G}_{m}^{|i|}$. Thus we may assume that $\mathcal{U}$ itself is an open subscheme of $\mathcal{G}=\mathrm{G}_{m}^{N}$ for some $N$. From the de- 
composition

$$
|\mathcal{G}|=\coprod_{n=1}^{\infty}\left|\mathcal{G}_{n}^{*}\right|
$$

we obtain

$$
|\mathcal{U}|=\coprod_{n=1}^{\infty}\left|\mathcal{U} \cap \mathcal{G}_{n}^{*}\right|
$$

and hence

$$
\zeta_{\mathcal{U}}(s)=\prod_{n=1}^{\infty} \zeta_{\mathcal{U} \cap \mathcal{G}_{n}^{*}}(s)
$$

Next, note that

$$
\mathcal{G}_{n}^{*}=\coprod_{j \in J_{n}} \mu_{j_{1}}^{*} \times \ldots \times \mu_{j_{N}}^{*}
$$

where $J_{n}$ is the set of $N$-tuples $j=\left(j_{1}, \ldots, j_{N}\right)$ of divisors of $n$ whose smallest common multiple is $n$. Thus

$$
\zeta_{\mathcal{U} \cap \mathcal{G}_{n}^{*}}(s)=\prod_{j \in J_{n}} \zeta_{\mathcal{U} \cap\left(\mu_{j_{1}}^{*} \times \ldots \times \mu_{j_{N}}^{*}\right)}(s) .
$$

We have seen that $\mu_{v}^{*}=\operatorname{spec} Z\left[1 / v, \zeta_{v}\right]$ where $\zeta_{v}$ is a primitive $v$-th root of unity. This gives

$$
\mathcal{U} \cap\left(\mu_{j_{1}}^{*} \times \ldots \times \mu_{j_{N}}^{*}\right)=\mathcal{U} \otimes_{\mathrm{Z}} R_{j}
$$

where

$$
R_{j}=Z_{[}\left[\zeta_{j_{1}}\right] \otimes_{Z} \ldots \otimes_{\mathrm{Z}} Z_{Z}\left[\zeta_{j_{N}}\right] \otimes_{\mathrm{Z}} \mathrm{Z}[1 / n]
$$

is an étale $Z[1 / n]$-algebra. We have

$$
\operatorname{spec} R_{j}=\coprod_{\alpha} \operatorname{spec} \mathfrak{D}_{K_{\alpha}}[1 / n]
$$

where $\alpha$ runs over the maximal ideals of $Q\left(\zeta_{j_{1}}\right) \otimes \ldots \otimes Q\left(\zeta_{j_{N}}\right)$. They correspond to the orbits of the diagonal $G_{\mathrm{Q}}$-action on $\mu_{j_{1}}^{*}(\overline{\mathrm{Q}}) \times \ldots \times \mu_{j_{N}}^{*}(\overline{\mathrm{Q}})$. It follows that

$$
\zeta_{\mathcal{U} \cap\left(\mu_{j_{1}}^{*} \times \ldots \times \mu_{j_{N}}^{*}\right)}(s)=\prod_{\alpha} \zeta_{\mathcal{U}_{\alpha}}(s)
$$

where $\mathcal{U}_{\alpha}=\mathcal{U} \cap \operatorname{spec} \mathfrak{D}_{K_{\alpha}}[1 / n]$ is an open subscheme of spec $\mathfrak{D}_{K_{\alpha}}[1 / n]$. Hence, up to finitely many Euler factors, $\zeta_{\mathcal{U}_{\alpha}}(s)$ is the Dedekind zetafunction $\zeta_{K_{\alpha}, S_{n}}(s)$. Thus, combining formulas (30)-(33), we obtain the desired horizontal factorization of $\zeta_{\mathcal{U}}(s)$. 
Acknowledgments. The first author would like to thank Dinakar Ramakrishnan for having shown him the infinite product formula in Taniyama's work many years ago. We also thank the referee for suggesting the statement and proof of proposition 1.

\section{REFERENCES}

[A] Tom M. Apostol, Resultants of cyclotomic polynomials, Proc. Amer. Math. Soc. 24 (1970), pp. 457-462.

[C] BRIAN CONRAD, A modern proof of Chevalley's theorem on algebraic groups, J. Ramanujan Math. Soc. 17 (1) (2002), pp. 1-18.

[DG] Michel Demazure - Pierre Gabriel, Groupes algébriques. Tome I: Géométrie algébrique, généralités, groupes commutatifs, Masson \& Cie, Éditeur, Paris, 1970. Avec un appendice Corps de classes local par Michiel Hazewinkel.

[EGAIV3] A. GROTHENDIECK, Éléments de géométrie algébrique. IV. Étude locale des schémas et des morphismes de schémas. III, Inst. Hautes Études Sci. Publ. Math., 28 (1966), p. 255.

[JR] Kirti Joshi - Ravi Raghunathan, Infinite product identities for Lfunctions, Illinois J. Math., 49 (3) (2005), pp. 885-891 (electronic).

[JY] Kirti Joshi - C. S. Yogananda, A remark on product of Dirichlet $L$ functions, Acta Arith., 91 (4) (1999), pp. 325-327.

[KL] HENRY H. KIm - KYU-HwAN LeE, Representation theory of p-adic groups and canonical bases, Adv. Math., 227 (2) (2011), pp. 945-961.

[L] QING LIU, Algebraic geometry and arithmetic curves, volume 6 of Oxford Graduate Texts in Mathematics, Oxford University Press (Oxford, 2002). Translated from the French by Reinie Erné, Oxford Science Publications.

[N] JÜRgen NEUKIRCH, Algebraic number theory, volume 322 of Grundlehren der Mathematischen Wissenschaften [Fundamental Principles of Mathematical Sciences] (Springer-Verlag, Berlin, 1999) Translated from the 1992 German original and with a note by Norbert Schappacher, with a foreword by G. Harder.

[S] JEAN-PIERRE SERre, Abelian l-adic representations and elliptic curves, McGill University lecture notes written with the collaboration of Willem Kuyk and John Labute. W. A. Benjamin, Inc., New YorkAmsterdam, 1968.

[T] YUTAKA TANIYAMA, L-functions of number fields and zeta functions of abelian varieties., J. Math. Soc. Japan, 9 (1957), pp. 330-366.

[Wa] William C. Waterhouse, Introduction to affine group schemes, volume 66 of Graduate Texts in Mathematics (Springer-Verlag, New York, 1979).

[We] ANDRÉ WEIL, Variétés abéliennes et courbes algébriques, Actualités Sci. Ind., no. 1064 = Publ. Inst. Math. Univ. Strasbourg 8 (1946), Hermann \& Cie., Paris, 1948.

Manoscritto pervenuto in redazione l'11 Febbraio 2012. 